\title{
Germanica
}

\section{L’Esthétique de la laideur de Karl Rosenkranz}

Karl Rosenkranz' Ästhetik des Hässlichen

\section{Norbert Waszek}

\section{(2) OpenEdition}

Journals

\section{Édition électronique}

URL : http://journals.openedition.org/germanica/466

DOI : 10.4000/germanica.466

ISSN : 2107-0784

Éditeur

Université de Lille

\section{Édition imprimée}

Date de publication : 1 décembre 2005

Pagination : 17-28

ISBN : 2-913857-16-7

ISSN : 0984-2632

Référence électronique

Norbert Waszek, "L'Esthétique de la laideur de Karl Rosenkranz », Germanica [En ligne], 37 | 2005, mis en ligne le 07 janvier 2010, consulté le 06 octobre 2020. URL : http://journals.openedition.org/ germanica/466 ; DOI : https://doi.org/10.4000/germanica.466

Ce document a été généré automatiquement le 6 octobre 2020.

(c) Tous droits réservés 


\title{
L'Esthétique de la laideur de Karl Rosenkranz
}

\author{
Karl Rosenkranz' Ästhetik des Hässlichen
}

Norbert Waszek

\section{Introduction}

1 Longtemps négligé en France, Karl Rosenkranz compte pourtant indubitablement parmi les grands maîtres universitaires du dix-neuvième siècle ${ }^{1}$. Né le 23 avril 1805 à Magdebourg, il fut, à partir de 1833, l'un des successeurs de Kant à la chaire de philosophie à l'Université de Königsberg, ville dans laquelle il mourut le 14 juin 1879. Nous devons à cet éminent historien de la philosophie des centaines de publications, parmi lesquelles des travaux importants sur Diderot, Kant et tout particulièrement Hegel $^{2}$ - ainsi qu'une œuvre plus originale : l'Esthétique du laid, qu'il publia en allemand en 1853 et dont la première traduction française, faite par Sibylle Muller plus de centcinquante ans après la première édition allemande, vient de sortir, précédée d'une présentation fouillée et fort utile, par Gérard Raulet ${ }^{3}$.

2 Dans la seconde moitié du dix-huitième siècle, la thèse si longtemps reconnue d'une unité du Beau, du Bon et du Vrai, est progressivement abandonnée. À en croire Rosenkranz, c'est alors la "philosophie allemande» qui «peut se glorifier d'être la première à avoir eu le courage de reconnaître le laid»(EL, p. 42). De cette reconnaissance, Rosenkranz donne comme exemple le plus précoce Lessing qui, dans son Laokoon (Chapitres XXIII à XXV), traita en effet du laid et du repoussant. Si la démarche de Rosenkranz ne choque plus guère le lecteur en ce début du XxI siècle, c'est peut-être en effet parce qu'elle s'annonce déjà chez Lessing. Comme l'écrit fort justement Michael Pauen :

À l'heure actuelle, les temps ont profondément changé. Parallèlement à d'autres phénomènes suscitant l'aversion, le laid s'est assuré un solide statut dans la pratique esthétique et il a ainsi perdu beaucoup de son potentiel subversif. Les théoriciens ont conclu la paix avec les « arts qui ne sont plus beaux » et même dans 
les feuilletons des journaux locaux, on accueille avec compréhension et sympathie

les entreprises de provocation esthétique ${ }^{4}$.

L'attitude communément adoptée aujourd'hui ne devrait néanmoins pas faire oublier que, dans la réévaluation philosophique du laid, Rosenkranz fut un pionnier. Il pouvait, certes, prendre appui sur quelques phrases de son maître Hegel, et plus particulièrement sur le Système de l'esthétique ${ }^{5}$ de Christian Hermann Weisse (1801-1866) - nous reviendrons encore dans la suite de cet article sur ces deux sources d'inspiration. Mais il fut le premier à placer le laid au centre d'un ouvrage de grande envergure, et à lui consacrer une analyse encyclopédique, c'est-à-dire ici systématique. Les premiers lecteurs de Rosenkranz furent d'ailleurs assez irrités par son œuvre. Certains n'étaient pas même prêts à en accepter le titre, perçu comme une contradiction: si en effet, selon la définition du père fondateur de la discipline, Alexander Gottlieb Baumgarten (1714-1762), l'esthétique est la théorie du beau, comment donc, se demandaient-ils, pourrait-il y avoir une esthétique de ce qui est contraire au beau? Une génération après, Karl August Varnhagen von Ense (1785-1858) lui-même, pourtant si généreux, resta perplexe lorsqu'il reçut le livre : pourquoi diable ne traiter que du laid, isolé du beau, semblait-il se demander - exprimant toutefois ses doutes de la façon suivante, plus feutrée et diplomatique :

Pourquoi considérer [le laid] à part? Pourquoi ne pas l'intégrer à l'ensemble de l'esthétique, où cette partie trouvera d'elle-même sa place, sa vraie place ? Traiter un objet à part suscite toujours le sentiment d'un manque ${ }^{6}$.

4 Il conviendra, dans les pages qui suivent, d'expliquer plus en détail le projet novateur et influent de Rosenkranz (II). Nous replacerons ensuite ce projet dans son contexte, l'histoire de l'esthétique en Allemagne (III) ; et nous ferons ressortir les implications que peut avoir ce projet pour ce qui concerne la critique sociale et politique (IV). Mais compte tenu de l'oubli relatif dans lequel, jusqu'à tout récemment, Rosenkranz est tombé en France, il nous faut d'abord brièvement rappeler quelques éléments de son parcours intellectuel (I).

\section{Karl Rosenkranz : « romantique défroquéę $\overline{F \theta}_{\theta \theta}$ » ou " hégélien pur beurre »?}

5 Nous fêtons en 2005 le bicentenaire de la naissance de Rosenkranz, qui mérite largement que le public se souvienne de son œuvre. Certes, il se dévalorise parfois luimême, par exemple lorsqu'il se décrit comme un épigone (Raulet cite un passage éloquent à cet égard, in $E L$, p. 8), mais c'est parce qu'il se compare avec Hegel, et avec Fichte : appliquer un standard aussi sévère réduirait tout le monde, ou presque, au statut d'épigones! Nous reviendrons dans la dernière partie de cet article sur l'engagement politique de Rosenkranz. Ici, il s'agit surtout de clarifier le destin intellectuel de notre auteur, par le biais de ses origines. En 1824, à l'âge de dix-neuf ans, Rosenkranz commença ses études dans la jeune mais déjà proéminente Université de Berlin, qu'il ne quitta qu'en 1826 pour poursuivre dans une autre université, celle de Halle, se conformant par là aux mœurs de la vie étudiante de l'époque en Allemagne.

6 À Berlin, Hegel était alors au faîte de sa gloire. Il attirait de plus en plus d'étudiants et de disciples. Si l'on pense à ce succès incontestable de Hegel à Berlin, ainsi qu'à l'hégélianisme ultérieur de Rosenkranz lui-même, il est plutôt surprenant de constater que notre auteur ne se soit pas immédiatement tourné vers cette philosophie, se 
ralliant par là aux nombreux auditeurs, souvent enthousiastes, qu'avait alors le philosophe. Ce fait nécessite d'autant plus une explication que Rosenkranz suivit bien les cours que proposait Leopold von Henning (1791-1866), en guise d'introduction à l' Encyclopédie de Hegel. Dans son autobiographie, Rosenkranz donne lui-même deux raisons pour expliquer l'incohérence dans son attitude, qui fit qu'il ne passa pas de l'introduction à Hegel faite par Henning au cours magistral du philosophe lui-même : il décrit d'abord, ironisant sur lui-même, les débuts de ses études, ce "mélange chaotique " (chaotisches Durcheinander) ${ }^{7}$ - on trouve déjà là les germes mêmes de ce talent encyclopédique, qui devait marquer son œuvre ultérieure ${ }^{8}$. La seconde raison est plus pertinente. Elle tient à son lien étroit avec l'un des grands concurrents et ennemis de Hegel, l'éminent théologien, philologue, mais aussi philosophe que fut Friedrich Schleiermacher (1768-1834). Rosenkranz fut, au début de ses études, complètement sous l'influence de celui-ci, que l'on peut considérer comme son premier vrai maître. Schleiermacher, qui était un brillant orateur, excellait comme professeur et prédicateur, surtout lorsqu'on le comparait à Hegel qui, selon le témoignage de Rosenkranz - un témoignage d'ailleurs conforme à celui de beaucoup de contemporains - avait plus de mal dans ses cours; et l'argument dut jouer pour le jeune étudiant qu'était alors Rosenkranz. Ce fut probablement une certaine loyalité envers Schleiermacher, dont il était un auditeur fidèle et un grand admirateur, qui poussa Rosenkranz à rester surtout avec celui-ci, et à ne s'aventurer que très rarement dans la salle de cours de Hegel. Les positions adoptées par Schleiermacher, mais aussi par son réseau de collègues et amis (parmi lesquels, pour ne citer qu'un nom, Friedrich Schlegel occupait une place de choix), conduisirent Rosenkranz à pénétrer, sans même peut-être le vouloir consciemment, dans l'épicentre du romantisme. Cette atmosphère dut marquer ses goûts et options littéraires, et elle ne sera pas sans conséquence pour son projet ultérieur, l'Esthétique du laid.

7 Il importe néanmoins de ne pas exagérer l'importance de cette première orientation de Rosenkranz, car elle fut en fin de compte éphémère - Rosenkranz n'en était qu'au début de ses études et il était encore très jeune. Il faut aussi regarder de plus près la façon dont Rosenkranz lui-même présente son engagement hégélien, car il est en tout cas difficile de mettre son propre témoignage en doute. Dans son autobiographie, notre auteur explique que, dès son premier séjour à Berlin et grâce au cours qu'il écouta chez von Henning, s'introduisirent en lui, sans qu'il en soit conscient ou y fasse attention, "l'étincelle de la démarche dialectique et le concept d'esprit », qui conditionnèrent ensuite le caractère de toute sa vie ${ }^{9}$. Ce fut donc un hégélien de la première heure, Hermann F.W. Hinrichs (1794-1861) ${ }^{10}$, qui, dès son arrivée à l'Université de Halle (en 1826), le gagna définitivement à la philosophie de Hegel. Rosenkranz date de cette époque son émancipation du "labyrinthe du romantisme", vers la "liberté de la philosophie $»^{11}$. Un séjour à Heidelberg (en 1827), où il fréquenta les cours d'un éminent théologien hégélien, Karl Daub (1765-1836), le confirma dans ce choix. Ce fut ensuite à cause de Hegel que, pendant l'automne de 1828, Rosenkranz retourna à Berlin. Dans ses propres publications, qui se succèdent de façon régulière et soutenue dès la fin des années 1820 , il se montre comme un défenseur constant et efficace de Hegel, qui ne s'abaisse jamais à une quelconque apologie idolâtre du maître ${ }^{12}$. En 1836, il rompt publiquement avec Schleiermacher, lorsqu'il publie la critique qu'il a faite de sa théologie ${ }^{13}$. Ce sera ensuite tout naturellement que la famille et les amis de Hegel lui confieront la rédaction de la biographie du philosophe, qui devait compléter la première édition complète de ses œuvres : G.W.F. Hegels Leben (1844). L'ouvrage, pour la 
composition duquel Rosenkranz eut accès à un grand nombre de manuscrits inédits de Hegel, ainsi qu'à des documents en possession de la famille, est un hommage appuyé à Hegel, mais sans hagiographie exagérée. Rosenkranz, dont pourtant certaines publications furent plus originales - comme par exemple l'Esthétique du laid, déjà citée considéra toujours ses travaux sur Hegel comme ceux qui exprimaient le meilleur de lui-même, et sa grande biographie de 1844 comme le livre qui resterait de lui :

Le Hegel que j'ai décrit dans cette biographie est le vrai Hegel, tel qu'il continuera à survivre dans les siècles à venir comme figure historique. Tout le reste de mes écrits peut sombrer dans l'oubli, mais la postérité retiendra de moi cette œuvre-là ${ }^{14 .}$

Quelle meilleure preuve pourrait-il y avoir de l'exactitude de cette prévision, que la récente traduction en français (cf. ci-dessus, note 2) de sa Vie de Hegel ?

\section{La descente en enfer}

9 L'expression qui sert de titre à cette section est un raccourci d'une phrase utilisée par Rosenzweig lui-même : «L'enfer n'est pas seulement religieux ou éthique, c'est aussi un enfer esthétique. [...] C'est dans cet enfer du beau que nous voulons descendre. » (EL, p. 41 - « Die Hölle ist nicht bloss eine religiös-ethische, sie ist auch eine ästhetische. [...] In diese Hölle des Schönen wollen wir hier niedersteigen. » ÄH, S. 11) Par ce parallèle entre l'enfer éthique et l'enfer esthétique, Rosenkranz s'intègre dès le départ dans une longue tradition, remontant jusqu'à l'Antiquité - on pensera par exemple au personnage de Thersite dans l'Iliade d'Homère - qui associe la laideur physique à la monstruosité morale. Manifestement, Rosenkranz reste néanmoins encore proche de l'idéal classique du beau, dans la mesure où pour lui aussi, comme dans la perspective classique, le laid n'est rien d'autre que le «beau négatif » (EL, p. 35 ; das Negativschöne ÄH, S. 5) : « La pure image du beau ressortirait avec d'autant plus d'éclat sur la sombre toile de fond du laid. » (EL, p. 66 - «Von der dunklen Folie des Hässlichen hebe sich das reine Bild des Schönen um so leuchtender ab. » ÄH, S. 36). L'originalité de l'Esthétique du laid réside plutôt dans le fait que Rosenkranz entend traiter de tout l'univers du laid, alors que, comme il le dit lui-même, d'autres «jusqu'ici » n'ont étudié «le concept du laid [que...] de façon éparse et secondaire» (EL, p. 36 - «der Begriff des Hässlichen bisher nur teils zerstreut und nebenbei [...] abgehandelt worden ist. » $\ddot{H}$, S. 6). Mais chez lui, même si le laid n'est jamais perçu que comme l'ombre, la toile de fond sombre du beau, il est devenu « inséparable » (unzertrennlich) du beau, et donc indispensable. De plus, le laid n'est pas seulement ou simplement, pour lui, le contraire du beau : ce n'est pas un concept statique, mais plutôt un concept qu'il faut saisir " comme en devenir " (EL, p. 168 - «als ein Werdendes» $\ddot{H} H$, S. 141). Pour reprendre ici l'heureuse formulation de Gérard Raulet dans sa préface à l'Esthétique du laid, la laideur est devenue « un moment de la réalisation de l'Idée esthétique » (EL, p. 12).

Si, d'ordinaire, la descente en enfer est présentée comme une sanction imposée suite aux péchés de l'homme, la descente de Rosenkranz dans l'enfer du beau est présentée au contraire comme volontaire : «nous voulons [y] descendre.» (EL, p. 41 - « wollen wir hier niedersteigen. » $\ddot{H}$, S. 11). L'auteur de l'Esthétique du laid explique cet acte volontaire par une référence curieuse à Charles Fourier (1772-1837) - il s'avère que de cette manière hégélianisme et proto-socialisme utopique se touchent - à savoir comme l'un de ces «travaux de dévouement» (en français dans le texte), auquel «certains hommes se résignent, parce qu'ils reconnaissent qu'il est nécessaire pour le bien 
commun"; ajoutant: "c'est à une obligation semblable que nous avons tenté de satisfaire. » (EL, p. 37 - « zu denen sich aber Menschen aus Resignation entschliessen, weil sie die Notwendigkeit derselben für das Gesamtwohl erkennen. Solch einer Pflicht ist auch hier zu genügen versucht worden. » $\ddot{H}$, S. 6) Mais, pourrait-on demander, le lecteur potentiel doit-il lui aussi se plonger dans l'étude d'un objet aussi repoussant ? "Sans aucun doute", répond Rosenkranz, car la recherche esthétique ne cesse de s'occuper de la question du laid depuis quelques temps (EL, p. 36 - «Unzweifelhaft » $\ddot{H} H$, S. 6). Pour exprimer cet avis de Rosenkranz de façon plus moderne, on pourrait dire que, lorsque Rosenkranz publia son Esthétique du laid, le temps était suffisamment mûr pour une reconnaissance esthétique du laid. Ce que ceci signifie pour le contexte esthétique d'une part, sociopolitique de l'autre, devra être clarifié dans les deux sections suivantes.

11 Partant de ces réflexions, Rosenkranz élabore alors, dans son ouvrage, une phénoménologie complète et une classification du laid - ou encore, pour reprendre la façon dont il s'exprime lui-même : «C'est en quelque sorte tout l'univers du laid que je déploie, depuis ses premières nébuleuses chaotiques, depuis l'amorphie et l'asymétrie, jusqu'à ses formations les plus denses dans l'infinie diversité de la désorganisation du beau qu'est la caricature. L'absence de forme, l'incorrection et la difformité constituent les différents degrés de cette série cohérente de métamorphoses.» (EL, p. 36 - «Ich rolle gleichsam den Kosmos des Hässlichen auf von seinen ersten chaotischen Nebelflecken, von der Amorphie und Asymmetrie an bis $\mathrm{zu}$ seinen intensivsten Formationen in der unendlichen Mannigfaltigkeit der Desorganisation des Schönen durch die Karikatur. Die Formlosigkeit, die Inkorrektheit und die Deformität der Verbildung machen die verschiedenen Stufen dieser in sich konsequenten Reihe von Metamorphosen aus. » $\ddot{H} H$, S. 5) Rosenzweig déploie aussi cet univers avec une grande richesse d'exemples, recherchés - pendant quinze ans (!), à en croire son propre témoignage - «dans tous les arts et dans toutes les époques 》 (EL, p. 36 - «Alle Künste und alle Epochen » $\ddot{H} H$, S. 6). Les très nombreux exemples dont il se sert semblent par ailleurs l'avoir troublé ${ }^{15}$ : tout se passe comme si ce grand poids empirique avait écrasé, en quelque sorte, la réflexion philosophique. En réalité, en tant que bon hégélien, Rosenkranz est ici tout à fait dans son droit : la philosophie hégélienne n'est pas une "spéculation » a priori qu'il faudrait confirmer ou corriger à l'aide de l'expérience. C'est une pensée nourrie de l'expérience, qui ne prend son envol que sur sa base ${ }^{16}$.

Chez Rosenkranz, le laid occupe en fin de compte, dans l'esthétique, la place de «moyen terme, entre le concept du beau et celui du comique» (EL, p. 35 - « die Mitte zwischen dem Schönen und dem Komischen.» $A ̈ H, ~ S .7)$. Le point peut-être le plus intéressant, dans cette perspective, concerne la façon dont le laid devient comique ( $E L$, p. 170 s). La forme de laid étudiée ici est la caricature, cette défiguration de l'idéal, qui devient comique : « La caricature exagère un caractère particulier au-delà de la mesure, produisant ainsi une disproportion et, en rappelant son contraire idéal, elle devient comique.» (EL, p. 172 - «Die Karikatur treibt ein Besonderes über das Mass hinaus, erzeugt dadurch ein Missverhältnis und wird, indem es an ihr ideales Gegenteil erinnert, komisch.» $A ̈ H, S .145)$ L'une des clés permettant de comprendre ce tournant se trouve probablement dans le rapport particulier établi entre l'idéal du beau et la liberté : la non-liberté, dans laquelle résident le laid et sa forme singulière, la caricature, "n'est pas simple absence de liberté », mais plutôt « la négation positive de la vraie liberté » (EL, p. 174 - «Die Unfreiheit ist nicht eine blosse Abwesenheit der Freiheit, sondern positive Negation der wirklichen Freiheit.» ÄH, S. 146). C'est ce libre 
choix de la négation de la liberté qui « fait du caractéristique un absolu [...] elle se coupe ainsi de l'idéal » et «c'est cette contradiction qui donne au spectateur matière à rire.» (EL, p. 175) - «verabsolutiert das Charakteristische [...], entzweit sich dadurch mit dem Ideal [...] und gewährt durch solchen Widerspruch dem Anschauenden Stoff zum Lachen. » (ÄH, S. 147)

\section{Le destin d'un pionnier : les contemporains le jugent trop audacieux, la postérité trop timide}

13 Pour comprendre la place de l'Esthétique du laid dans la pensée esthétique allemande de l'époque, il convient d'étudier les pistes de recherche importantes ouvertes par l'auteur lui-même. Nous avons déjà évoqué la tradition à laquelle il se rattache : inaugurée par Lessing ( $E L$, p. 377, note 1), elle insère l'examen du laid dans le cadre d'une réflexion esthétique plus vaste. Mais si, dès le XviII ${ }^{\mathrm{e}}$ siècle, le laid avait déjà attiré quelque attention, l'intérêt éveillé par le sujet était resté limité et ponctuel : la réflexion visait seulement l'explication de quelques personnages 'bizarres' de Shakespeare, Goethe ou Byron (EL, p. 42). C'est Ch.H. Weisse, cité à plusieurs reprises dans son texte (EL, par exemple, p. 168, 316 s), que Rosenkranz salue et apprécie, pour avoir attribué au laid une place systématique dans la pensée esthétique : «Le mérite d'avoir consciemment introduit dans la science le concept du laid comme moment organique de l'idée du beau revient à Chr. H. Weisse » (EL, p. 377 - «Das Verdienst, den Begriff des Hässlichen als ein organisches Moment der Idee des Schönen mit Bewusstsein in die Wissenschaft eingeführt zu haben, gebührt Chr. H. Weisse » ÄH, S. 345). Weisse, en effet, considéra le laid aussi bien que le beau comme une émanation de l'Esprit Absolu (il reprend ainsi la terminologie hégélienne) ${ }^{17}$. Il ne se serait même pas limité à cette réussite d'ordre fondamental : il aurait même anticipé la conception du laid comme « en devenir », en d'autres termes comme moment, dans la dialectique de la réalisation de l'Idée esthétique. Il est moins aisé de détecter ce que Rosenkranz reproche à Weisse, et en quoi il voudrait aller plus loin : il semble d'abord le considérer comme l'un de ceux qui en sont restés « tantôt à une réflexion générale, courte et peu approfondie, tantôt à une conception spiritualiste trop partielle ${ }^{18}$. » Il lui reproche aussi, parfois, des faiblesses dans la présentation, parlant de «Kühnheit » (audace; EL, p. 168) et de «Verirrung » ( égarement; EL, p. 317).

14 L'hégélianisme de Rosenkranz, le fait que Weisse lui-même soit resté dans le sillage de Hegel (malgré quelques efforts pour s'en libérer) nous obligent à un commentaire sur le statut du laid dans son Esthétique. À première vue, le classicisme bien connu de Hegel les sculptures de l'Antiquité grecque restent le grand modèle de la conception hégélienne du beau - ne semble guère laisser de place au laid. Pour Hegel, l'art est (avec la religion et la philosophie) une partie intégrante de l'esprit absolu: sa fonction par excellence ne peut donc être que celle d'exprimer la vérité, et il semble bien que le laid et le mensonge doivent être éliminés de la systématique philosophique. À y regarder de plus près, pourtant, on trouve tout de même, dans l'appréciation d'un article d'Aloys Hirt (1759-1839) au centre duquel se trouve le concept de caractéristique, quelques phrases qui semblent admettre le laid et la représentation du laid, ou du moins leur laisser une petite porte ouverte - car la caricature reste en rapport avec celui-ci, même si elle est "un excès de caractéristique $»^{19}$. De plus, et voilà qui est sans aucune commune mesure plus important que quelques phrases formulées en passant, Hegel 
revient implicitement sur le laid à la fin de son traitement de l'art romantique. Rappelons que l'art, selon Hegel, passe d'abord par les formes symboliques et classiques, et qu'il ne survit dans le romantisme que sous une forme réduite. Il s'agit là d'une décadence de l'art qui libère la subjectivité de sa contingence sous la forme de l'humour. Avec l'humour comme façon d'assumer le laid, et de se sauver ainsi de la simple négation du beau, Hegel et Rosenkranz se trouvent à nouveau très proches l'un de l'autre. Par son analyse de l'art romantique, Hegel fournit en effet, en germe, quelques éléments puissants et influents d'une esthétique de l'art moderne. Cette analyse, dont la signification ne fut redécouverte qu'au vingtième siècle, ne constitue pourtant pas encore une théorie esthétique explicite et complète du laid, et c'est peutêtre cette ambivalence qui explique pourquoi Rosenkranz, qui fut un fidèle et brillant interprète de Hegel (cf. ci-dessus, section II), n'accorde pas plus de place à la discussion avec Hegel dans son Esthétique du laid, ce document majeur de l'esthétique posthégélienne.

15 L'ouvrage de Rosenkranz subit ensuite le sort si souvent réservé par le destin aux authentiques pionniers: être ridiculisé deux fois, en premier lieu par les contemporains, puis par la postérité. Si, comme nous l'avons indiqué dès l'introduction, les lecteurs de l'époque ne pouvaient qu'être choqués, ou irrités, par la vaste phénoménologie du laid de Rosenkranz - ils étaient encore trop figés dans une esthétique de l'art apollinien - au vingtième siècle, ses lecteurs jugèrent son identification du laid à la faiblesse morale datée, et dépassée. La définition du laid comme «beau négatif » par laquelle Rosenzweig ouvre son analyse, ne leur semble pas permettre une considération du laid comme une véritable catégorie, une catégorie esthétique de plein droit - ce que l'on exige aujourd'hui ${ }^{20}$.

\section{L'actualité de l'Esthétique du laid révélée par Adorno}

16 En conclusion de cet article, une réflexion sur l'actualité de Rosenkranz me semble appropriée: son entreprise semble bien avoir préservé une fonction critique. Rappelons à ce sujet la phrase terrible de Henri Lefebvre (1905-1991) dans sa Critique de la vie quotidienne - une phrase qui se fixe dans la mémoire : «La beauté s'est figée en froides pièces de musée surnageant dans l'océan boueux de la misère ${ }^{21}$. » À l'arrièrefond de cette réflexion se trouve la conviction que le laid, dévoilé sans idéalisation, peut devenir libérateur, et contribuer ainsi à la critique d'un monde devenu " moche ». Parce que - pour Rosenkranz autant que, déjà, pour Plotin -, la laideur physique fut associée à la monstruosité morale, une telle critique ne se réduit pas à un débat esthétique, mais s'élargit au contraire, pour se transformer en une arme dans la lutte contre une réalité sociale devenue insupportable. Le terme d'origine argotique " moche », que nous venons d'utiliser et qui est l'un des synonymes français de « laid», préserve d'ailleurs à merveille cette double connotation, physique et morale: une architecture ratée peut être qualifiée de "moche», tout autant qu'un licenciement abusif (méprisable). C'est, me semble-t-il, dans cette perspective critique que l' Esthétique du laid de Karl Rosenkranz pourrait être fort utile.

17 Il est vrai qu'une telle critique politique, ou de société, ne se trouve nulle part de façon explicite chez Rosenkranz lui-même. Mais si l'on prend la peine de lire, au-delà de la surface, entre les lignes de l'ouvrage, et de le replacer dans son contexte, le sens 
critique de l'entreprise devient évident. Ainsi, pour ce qui concerne d'abord le contexte dans lequel le livre fut rédigé, la plupart des commentateurs sont d'accord pour affirmer - et G. Raulet le rappelle dans sa présentation ( $E L$, p. $18 \mathrm{~s}$ ) - que l'Esthétique du laid fut aussi le produit de la frustration politique de son auteur. Rosenkranz, ce professeur installé à Königsberg, c'est-à-dire dans un centre du libéralisme du Vormärz, s'était fortement engagé politiquement en 1848/49. Il fut même appelé à Berlin : on songeait à lui comme ministre de l'éducation, et il servit en fait comme conseiller. Mais l'échec du mouvement politique auquel il s'était identifié le conduisit à se résigner, à se retirer de la politique et à se consacrer à l'esthétique. La direction qu'il impartit à son travail théorique trahit néanmoins à nouveau son engagement critique : il trouva la grande majorité de ses exemples de laideur - une véritable "galerie des horreurs " (EL, p. 19) - à sa propre époque - "parce qu'ils étaient bien sûr tout frais dans ma mémoire » (EL, p. 38 - « weil sie natürlich mir am frischesten im Gedächtnis waren » $\ddot{H}$ , S. 8), écrit-il dans sa préface, comme pour s'excuser. Mais il ne trompa personne de cette manière : le choix de ses exemples reflète clairement son attitude critique envers son temps, surtout après l'échec de la vague révolutionnaire de 1848. Ecrire des ouvrages comme l'Esthétique du laid, dira-t-il une fois dans un autre texte autobiographique - à bon entendeur salut ! - voilà qui était pour lui une façon de se «sauver des misères de la vie " ${ }^{22}$.

Lorsque T.W. Adorno (1903-1969), voulant expliquer les raisons stratégiques pour lesquelles il utilise lui-même le laid dans cette partie de sa Théorie esthétique qu'il intitule «Aspect social et philosophie de l'histoire du laid» ("Sozialer Aspekt und Geschichtsphilosophie des Hässlichen »), écrit que «L'art doit faire son affaire de ce qui est mis à l'index en tant que laid [...] pour dénoncer, dans le laid, le monde qui le crée et le reproduit à son image $»^{23}$ - il cite Rosenkranz comme l'un de ses prédécesseurs : "Quoiqu'il en soi, le laid doit constituer ou pouvoir constituer un moment de l'art. Esthétique du laid, tel est le titre d'une œuvre du disciple de Hegel, Rosenkranz ${ }^{24}$.» Ce fait ne fut guère apprécié jusqu'à présent dans la recherche sur Adorno.

\section{NOTES}

1. L'autobiographie de Rosenkranz - Von Magdeburg bis Königsberg. Berlin, Heimann, 1873 - couvre la période qui va jusqu'à sa nomination à la chaire de Kant; cf. Norbert Waszek : «Rosenkranz, Karl », in : Dictionnaire du XIX siècle européen. Ed. par Madeleine Ambrière. Paris, PUF, 1997, p. 1034 ; «Karl Rosenkranz als Hegelianer - Im Lichte seiner Briefe », in: Jahrbuch für Hegelforschung. Bd. 3 (1997), S. 287-294.

2. Depuis peu disponible en français: Karl Rosenkranz, Vie de Hegel [1844], suivi de : Apologie de Hegel contre le docteur Haym [1858], traduit par Pierre Osmo. Paris, Gallimard, 2004. La présentation détaillée de P.Osmo : «Karl Rosenkranz, un hégélien singulier » (p. 9-62) est fort instructive.

3. Karl Rosenkranz : Die Ästhetik des Hässlichen. Königsberg, Bornträger, 1853 (citée par la suite : ÄH, d'après l'édition suivante : Leipzig, Reclam, 1990) - Esthétique du laid [citée par la suite : EL], traduite par Sibylle Muller, présentée par Gérard Raulet. Paris, Circé, 2004. 
4. M. Pauen : «Die Ästhetik des Hässlichen », in : Information Philosophie. Bd. 32.3 (2004), S. 14 : «Mittlerweile haben sich die Zeiten [...] entscheidend geändert. Das Hässliche hat zusammen mit anderen aversiven Phänomenen einen festen Platz in der ästhetischen Praxis gefunden und damit viel von seiner provokativen Kraft eingebüsst. Die Theoretiker haben ihren Frieden mit den 'nicht mehr schönen' Künsten geschlossen und auch in den Feuilletons der Lokalzeitungen werden ästhetische Provokationsversuche mit freundlichem Verständnis entgegengenommen. » 5. Christian Hermann Weisse : System der Ästhetik als Wissenschaft von der Idee der Schönheit. 2 vols. Leipzig, Hartmann, 1830.

6. K.A. Varnhagen von Ense : "Brief an Karl Rosenkr²anz (vom 1. Juli 1853) ", in : Briefwechsel zwischen Karl Rosenkranz und Varnhagen von Ense. Ed. par Arthur Warda. Königsberg, Gräfe \& Unzer, 1926, p. 199 : «Warum diese Absonderung [des Hässlichen ; N.W.], warum nicht lieber die ganze Aesthetik, wo dieser Theil von selbst auch seine Stelle findet, seine richtige Stelle? Die abgesonderte Behandlung lässt immer etwas vermissen. »

7. K. Rosenkranz : Von Magdeburg bis Königsberg, op. cit., p. 185.

8. Cf. Ibid., p. 186 : "Meine allgemeine Bildung war eine sehr ausgedehnte, und es war mir eigentlich keine Wissenschaft ganz fremd geblieben, da ich [...] stark in die encyklopädische Zerstreuung gefallen war. " / "Ma culture générale était très étendue et aucune science ne m'était restée totalement étrangère, car je [...] m'étais fortement adonné au loisir encyclopédique. "

9. K. Rosenkranz: Von Magdeburg bis Königsberg, op. cit., p. 188: «so war der Funke der dialektischen Methode und der Begriff des Geistes still und unbewacht in meine Seele gefallen und glimmte im Verborgenen rastlos nagend weiter. Die ganze Signatur meines Lebens wurde in jenem fröhlichen Sommer [1825; N.W.] festgestellt ».

10. Hinrichs avait déjà été l'élève de Hegel à Heidelberg. Hegel lui avait fait l'honneur de rédiger une préface pour son étude : Die Religion im inneren Verhältnis zur Wissenschaft. Mit einem Vorwort von G.W.F. Hegel. Heidelberg, Groos, 1822. Hinrichs fut probablement le premier des disciples de Hegel à devenir lui-même professeur des universités, d'abord à Breslau (1822), puis à Halle (1824). 11. Cf. K. Rosenkranz : Von Magdeburg bis Königsberg, op. cit., p. VIII.

12. Cf. K. Rosenkranz: Hegel als deutscher Nationalphilosoph. Leipzig, Duncker \& Humblot, 1870, p. XII : « Nun hab' ich allerdings mir stets freien Sinn bewahrt, weil ich Sclaverei jeder Art hasse. Buchstabenvergötterung in der Philosophie ist der Tod derselben. »

13. K. Rosenkranz, Kritik der Schleiermacherschen Glaubenslehre. Königsberg, Unzer, 1836.

14. K. Rosenkranz: Hegel als deutscher Nationalphilosoph, op. cit., p. V : «Der Hegel, den ich in jener Biographie geschildert habe, ist der wirkliche Hegel, wie er, als historische Person, in die künftigen Jahrhunderte fortwandern wird. Alles andere, was ich geschrieben habe, kann der Vergessenheit zum Raube verfallen, aber dies Buch von mir wird bleiben. »

15. EL, p. 37: «Au cours de cet ouvrage, il m'est arrivé de m'excuser en quelque sorte de ma propension à penser par exemple.»- $\ddot{H}$, S. 7 : "Im Verlauf der Abhandlung habe ich mich einmal darüber gewissermassen entschuldigt, so viel in Beispielen zu denken. »

16. Le meilleur commentaire de ce point difficile de la philosophie de Hegel est fourni par Bernard Bourgeois dans cette partie de sa présentation à son édition de la Philosophie de l'Esprit de Hegel intitulée «L'Aristote des temps modernes » (Encyclopédie des sciences philosophiques. III. Paris, Vrin, 1988, p. 85-89).

17. Ch.H. Weisse : System der Ästhetik..., op. cit., p. 180.

18. $E L$, p. 42 - «teils bei einer kurzen, wenig eingehenden Allgemeinheit, teils bei einer zu einseitig spiritualistischen Fassung stehengeblieben» ÄH, S. 12 ; cf. EL, p. 377 : «Weisse avait de la non-idée du laid une conception trop spiritualiste » - «Weisse hatte jedoch die Unidee des Hässlichen [...] zu spiritualistisch gefasst » $\ddot{H}$, S. 345 .

19. G.W.F. Hegel : Cours d'esthétique I (Edition Hotho). Trad. par Jean-Pierre Lefebvre et Veronika von Schenk. Paris, Aubier, 1995, p. 26-29 - «ein Überfluss des Charakteristischen » Hegel, 
Vorlesungen über die Ästhetik I. Theorie Werkausgabe. Vol. 13, Frankfurt/Main, Suhrkamp, 1970, S. 35.

20. M. Pauen : «Die Ästhetik des Hässlichen», op. cit., p.19: «Die Konsequenz aus diesen Überlegungen kann nur sein, das Hässliche als eine Kategorie eigenen Rechts zu betrachten »«On ne peut que déduire de ces réflexions que le laid doit être considéré comme une catégorie de plein droit. »

21. Henri Lefebvre : Critique de la vie quotidienne. Paris, L'Arche, 1958, p. 53.

22. K. Rosenkranz: Aus einem Tagebuch. Königsberg Herbst 1833 bis Frühjahr 1846. Leipzig, Brockhaus, 1854, p. 48 : «Rettung aus den Jämmerlichkeiten des Lebens ».

23. Theodor Wiesengrund Adorno : Théorie esthétique. Trad. par Marc Jimenez. Paris, Klincksieck, 1974, p. 71 - Adorno : Ästhetische Theorie. Ed. par Gretel Adorno et Rolf Tiedemann. Frankfurt/ Main, Suhrkamp, 1970, S. 78 f: « Kunst muss das als hässlich Verfemte zu ihrer Sache machen [...], um im Hässlichen die Welt zu denunzieren, die es nach ihrem Bilde schafft und reproduziert ".

24. Théorie esthétique, p. 67 - Ästhetische Theorie, S. 74 : «Was immer es sei, soll es [das Hässliche] ein Moment der Kunst bilden oder bilden können ; ein Werk des Hegelschülers Rosenkranz trägt den Titel Ästhetik des Hässlichen.»

\section{RÉSUMÉS}

L'Esthétique du laid, que Karl Rosenkranz publia en allemand en 1853, vient de paraître en traduction française - juste à temps pour le bicentenaire de la naissance de son auteur. Après un court rappel de quelques éléments du parcours intellectuel de Rosenkranz (I), cet article présente et explique de façon plus détaillée le projet innovateur et influent de Rosenkranz (II), pour le replacer ensuite dans son contexte, l'histoire de l'esthétique en Allemagne, en reconstruisant plus particulièrement son rapport aux esthétiques de Ch.H. Weisse et de Hegel (III). Une dernière partie (IV) fait ressortir les implications critiques du projet, en revenant sur l'engagement politique de Rosenkranz, mais en montrant aussi en quoi il constitue une anticipation de certaines thèses développées dans la Théorie esthétique de T.W. Adorno.

Zum 200. Geburtstag von Karl Rosenkranz erschien erstmals eine französische Übersetzung seiner Ästhetik des Hässlichen (1853). Der vorliegende Aufsatz will diese Ästhetik in vier Schritten vorstellen. Nach einigen Bemerkungen zur intellektuellen Biographie von Rosenkranz (I), werden der systematische Ort, an den Rosenkranz das Hässliche im Rahmen seines ästhetischen Denkens stellt, und dessen enzyklopädische Behandlung aufgezeigt (II). Ein weiterer Abschnitt (III) geht dem Kontext des Werkes von Rosenkranz im Rahmen der deutschen Tradition ästhetischer Systembildungen nach, wobei den Vorgängern von Rosenkranz, Ch.H. Weisse und Hegel besondere Aufmerksamkeit gewidmet wird. Ein abschliessender Abschnitt (IV) verdeutlicht das kritische Potential des Ansatzes von Rosenkranz mit Hilfe eines Rückblickes auf sein politisches Engagement und eines Ausblicks auf die Funktion des Hässlichen in der -Ästhetischen Theorie Adornos. 
INDEX

Mots-clés : beauté, laideur

\section{AUTEURS}

\section{NORBERT WASZEK}

Université Paris VIII : Vincennes à Saint Denis 